\title{
NEW GENERALIZED RIEMANN-LIOUVILLE FRACTIONAL INTEGRAL INEQUALITIES FOR CONVEX FUNCTIONS
}

\author{
PSHTIWAN OTHMAN MOHAMMED
}

Abstract. In the literature, the right-side of Hermite-Hadamard's inequality is called trapezoid type inequality. In this paper, we obtain new integral inequalities of trapezoid type for convex functions involving generalized Riemann-Liouville fractional integrals ( $\psi$-Riemann-Liouville fractional integrals). Our obtained inequalities generalize some recent classical integral inequalities and Riemann-Liouville fractional integral inequalities which are established in earlier works.

Mathematics subject classification (2020): 26D07, 26D15, 26D10, $26 \mathrm{~A} 33$.

Keywords and phrases: Riemann-Liouville fractional integral, convex function, Hermite-Hadamard inequality, convex functions, trapezoid formula.

\section{REFERENCES}

[1] D. Baleanu, P. O. Mohammed, S. Zeng, Inequalities of trapezoidal type involving generalized fractional integrals, Accepted.

[2] P. Ciatti, M. G. Cowling, F. Ricci, Hardy and uncertainty inequalities on stratified Lie groups, Adv. Math. 277 (2015), pp. 365-387.

[3] S. S. Dragomir, C. E. M. Pearce, Selected topics on Hermite-Hadamard inequalities and applications, RGMIA Monographs, Victoria University, 2000.

[4] S. S. Dragomir, Hermite-Hadamard inequality for fractional integrals via $\eta$-convex functions, Acta Math. Univ. Comenianae LXXXVI(1) (2017), pp. 153-164.

[5] S. S. Dragomir, M. I. Bhatti, M. IQbal, M. Muddassar, Some new Hermite-Hadamard's type fractional integral inequalities, J. Comput. Anal. Appl. 18 (4) (2015), pp. 655-661.

[6] A. Fernandez, P. O. Mohammed, Hermite-Hadamard inequalities in fractional calculus defined using Mittag-Leffler kernels, Math. Meth. Appl. Sci. 2020, 1-18, https://doi.org/10.1002/mma.6188.

[7] B. Gavrea, I. Gavrea, On some Ostrowski type inequalities, Gen. Math. 18 (1) (2010), pp. 33-44.

[8] H. Gunawan, ERIDAni, Fractional integrals and generalized Olsen inequalities, Kyungpook Math. J. 49 (2009), pp. 31-39.

[9] M. A. Hanson, On sufficiency of the Kuhn-Tucker conditions, J. Math. Anal. Appl. 80 (1981), pp. $545-550$.

[10] A. A. Kilbas, H. M. SRivastava, J. J. Trujillo, Theory and Applications of Fractional Differential Equations, Elsevier B. V., Amsterdam, Netherlands, 2006.

[11] T. Lian, W. TANG, R. ZHou, Fractional Hermite-Hadamard inequalities for $(s, m)$-convex or $s$ concave functions, J. Inequal. Appl. 2018 (2018), 240.

[12] P. O. Монамmed, New integral inequalities for preinvex functions via generalized beta function, J. Interdiscip. Math. 22 (4) (2019), pp. 539-549.

[13] P. O. Mohammed, On New Trapezoid Type Inequalities for h-convex Functions via Generalized Fractional Integral, Turkish J. Anal. Number Theory 6 (4) (2018), pp. 125-128.

[14] P. O. Mohammed, Some new Hermite-Hadamard type inequalities for MT-convex functions on differentiable coordinates, J. King Saud Univ. Sci. 30 (2018), pp. 258-262.

[15] P. O. Монамmed, Inequalities of Type Hermite-Hadamard for Fractional Integrals via Differentiable Convex Functions, Turkish J. Anal. Number Theory 4 (5) (2016), pp. 135-139. 
[16] P. O. Монамmed, Inequalities of $(k, s),(k, h)$-Type For Riemann-Liouville Fractional Integrals, Appl. Math. E-Notes 17 (2017), pp. 199-206.

[17] P. O. Mohammed, Hermite-Hadamard inequalities for Riemann-Liouville fractional integrals of a convex function with respect to a monotone function, Math. Meth. Appl. Sci. (2019), pp. 1-11, https://doi.org/10.1002/mma.5784.

[18] P. O. Mohammed, T. Abdeljawad, Modification of certain fractional integral inequalities for convex functions, Adv. Differ. Equ. 2020 (2020), 69.

[19] P. O. Mohammed, I. BReVIK, A New Version of the Hermite-Hadamard Inequality for RiemannLiouville Fractional Integrals, accepted.

[20] P. O. Mohammed, F. K. Hamasalh, New Conformable Fractional Integral Inequalities of Hermite-Hadamard Type for Convex Functions, Symmetry 11 (2) (2019), 263, https://doi.org/10.3390/sym11020263.

[21] P. O. Mohammed, M. Z. SARIKAYA, Hermite-Hadamard type inequalities for $\digamma$-convex function involving fractional integrals, J. Inequal. Appl. 2018 (2018), 359.

[22] P. O. Mohammed, M. Z. SARIKAYA, On generalized fractional integral inequalities for twice differentiable convex functions, J. Comput. Appl. Math. 372 (2020), 112740.

[23] P. O. Mohammed, M. Z. Sarikaya, D. Baleanu, On the Generalized HermiteHadamard Inequalities via the Tempered Fractional Integrals, Symmetry 12 (4) (2020), 595, https://doi:10.3390/sym12040595.

[24] F. QI, P. O. Mohammed, J. C. YaO, Y. H. YaO, Generalized fractional integral inequalities of Hermite-Hadamard type for $(\alpha, m)$-convex functions, J. Inequal. Appl. 2019 (2019), 135.

[25] M. E. Ozdemir, M. Avci, H. Kavurmaci, Hermite-Hadamard-type inequalities via $(\alpha, m)$ convexity, Comput. Math. Appl. 61 (2011), pp. 2614-2620.

[26] J. PARK, Some Hermite-Hadamard type inequalities for MT-convex functions via classical and Riemann-Liouville fractional integrals, Appl. Math. Sci. 9 (101) (2015), pp. 5011-5026.

[27] M. Z. SARIKAYA, E. SET, H. YALdiz, N. BAŞAK, Hermite-Hadamard's inequalities for fractional integrals and related fractional inequalities, Math. Comput. Model. 57 (2013), pp. 2403-2407.

[28] Y. Sawano, H. WAdAde, On the Gagliardo-Nirenberg type inequality in the critical Sobolev-Morrey space, J. Fourier Anal. Appl. 19 (1) (2013), pp. 20-47.

[29] E. Set, M. E. Ozdemir, M. Z. Sarikaya, F. Karakoc, Hermite-Hadamard type inequalities for $(\alpha, m)$-convex functions via fractional integrals, Moroccan J. Pure and Appl. Anal. 3 (1) (2017), pp. $15-21$.

[30] J. V. C. Sousa, E. C. Oliveira, On the $\Psi$-Hilfer fractional derivative, Commun. Nonlinear Sci. Numer. Simul. 60, pp. 72-91.

[31] J. Wanga, X. Lia, M. Feckanb, Y. Zhou, Hermite-Hadamard-type inequalities for RiemannLiouville fractional integrals via two kinds of convexity, Applicable Analysis 92(11) (2013), pp. 22412253.

[32] A. WeIR, B. Mond, Preinvex functions in multiple objective optimization, J. Math. Anal. Appl. 136 (1988), pp. 29-38. 